\title{
About one extremal problem for open sets and partially non-overlapping domains
}

\author{
Andrey L. Targonskit, Irina I. Targonskaya, K. Vashenko
}

\author{
(Presented by O. Dovgoshey)
}

\begin{abstract}
Sharp estimates of product of inner radii for pairwise disjoint domains are obtained. In particular, we solve an extremal problem in the case of arbitrary finite number of free poles on the system points on the rays.
\end{abstract}

2010 MSC. Primary 30C70, 30C75.

Key words and phrases. Inner radius of domain, quadratic differential, piecewise-separating transformation, the Green function, radial systems of points, logarithmic capacity, variational formula.

\section{Introduction}

This paper belongs to the theory of extremal problems on classes of disjoint domains, which is a separate direction in the geometric theory of functions of a complex variable. The start of these investigations is associated with the paper by M. A. Lavrent'ev [1]. He found the maximum of some functional with respect to two simply connected domains with two fixed points. We note that he applied this result to some aerodynamics problems. In 1947, G. M. Goluzin solved a similar problem for three fixed points on the complex plane [2]. Then the topic began to evolve rapidly. In this connection, we may recall papers of many authors, including Y. E. Alenitsin, M. A. Lebedev, J. Jenkins, P. M. Tamrazov, P. P. Kufarev, and others. Using the idea of P. M. Tamrazov, G.P. Bakhtina solved, for the first time, the problem with the so-called "free poles" on a unit circle (see, e.g., [3]).

An important step for the development of this topic was the papers by V.N. Dubinin. He proposed a new method of piecewise separating

Received 01.05.2019

The author is grateful to Prof. Bakhtin for suggesting problems and useful discussions. 
transformation and first solved a number of extremal problems for any multiconnected disjoint domains (see, e.g., [4-6]). Now, this type of extremal problems is used in investigations in the holomorphic dynamics.

In the last decade, Bakhtin's method of "managing functional" is actively used. A. K. Bakhtin solved a number of extremal problems for the so-called "radial systems of points" (see, e.g., [4, 7-20]). Namely this method will be applied in what follows.

Let $\mathbb{N}$ and $\mathbb{R}$ be the sets of natural and real numbers, respectively, let $\mathbb{C}$ be the plane of complex numbers, and let $\overline{\mathbb{C}}=\mathbb{C} \bigcup\{\infty\}$ be the Riemannian sphere, $\mathbb{R}_{+}=(0 ; \infty)$.

For a fixed number $n \in \mathbb{N}$ of points

$$
A_{n}=\left\{a_{k}\right\}_{k=1}^{n},
$$

the following relations are valid:

$$
0=\arg a_{1}<\arg a_{2}<\ldots<\arg a_{n}<2 \pi
$$

For such systems of points, we consider the following sizes:

$$
\sigma_{k}=\frac{1}{\pi}\left(\arg a_{k+1}-\arg a_{k}\right), k=1,2, \ldots, n, \quad a_{n+1}:=a_{1} .
$$

Consider the system of angular domains

$$
M_{k}:=\left\{w: \arg a_{k}<\arg w<\arg a_{k+1}\right\}, \quad k=\overline{1, n}, \quad a_{n+1}:=a_{1},
$$

and the following "managing functional" for an arbitrary system of points $A_{n}$ :

$$
\mathrm{T}\left(A_{n}\right)=\prod_{k=1}^{n} \chi\left(\left|\frac{a_{k}}{a_{k+1}}\right|^{\frac{1}{2 \sigma_{k}}}\right)\left|a_{k}\right|,
$$

where $\chi(t)=\frac{1}{2}\left(t+\frac{1}{t}\right)$.

Let $D, D \subset \overline{\mathbb{C}}$, be any open set, and let the point $w=a \in D$. By $D(a)$, we denote a connected component of the set $D$, which contains the point $a$. For an arbitrary system of points $A_{n}=\left\{a_{k} \in \mathbb{C}: k=\overline{1, n}\right\}$, satisfying condition (1.1) and for the open set $D, A_{n} \subset D$, we denote, by $D_{k}\left(a_{s}\right)$, the connected component of the set $D\left(a_{s}\right) \bigcap \overline{M_{k}}$ containing the point $a_{s}, k=\overline{1, n}, s=k, k+1, a_{n+1}:=a_{1}$.

We say that the open set $D, A_{n} \subset D$, satisfies the condition of disjointness relative to the system of points $A_{n}$, if the relation

$$
D_{k}\left(a_{k}\right) \bigcap D_{k}\left(a_{k+1}\right)=\varnothing
$$


$k=\overline{1, n}$, on all angles $\overline{M_{k}}$, holds.

System domains $\left\{B_{k}\right\}_{k=1}^{n}, k=\overline{1, n}$, the define system partially nonoverlapping domains, if

$$
D:=\bigcup_{k=1}^{n} B_{k},
$$

is open set, if she meets a condition (1.2).

Let

$$
g_{B}(z, a)=h_{B, a}(z)+\log \frac{1}{|z-a|}
$$

be the generalized Green function of a domain $B$ relative to the point $a \in B$. If $a=\infty$, then

$$
g_{B}(z, \infty)=h_{B, \infty}(z)+\log \frac{1}{|z|} .
$$

The quantity

$$
r(B, a):=\exp \left(h_{B, a}(z)\right)
$$

stands for the internal radius of the domain $B \subset \overline{\mathbb{C}}$ relative to the point $a \in B$ (see $[4-6,16,17,21])$.

We use the concept of a quadratic differential. We note that the main results on the theory of quadratic differentials can be found in work [22].

In what follows, we consider the following problems.

Problem 1. Let $n \in \mathbb{N}, n \geq 2, \alpha \geq 0$. To find a maximum of the functional

$$
\prod_{k=1}^{n}\left(\left|a_{k+1}-a_{k}\right|^{\alpha} \cdot r\left(D, a_{k}\right)\right)
$$

where $A_{n}=\left\{a_{k}\right\}_{k=1}^{n}$ is any ray system of points that satisfies condition (1.1), $D$ is an open set that satisfies condition (1.2), $a_{k} \in D \subset \overline{\mathbb{C}}$, and to describe all extremals $(k=\overline{1, n})$.

Problem 2. Let $n \in \mathbb{N}, n \geq 2, \alpha \geq 0$. To find a maximum of the functional

$$
\prod_{k=1}^{n}\left(\left|a_{k+1}-a_{k}\right|^{\alpha} \cdot r\left(B_{k}, a_{k}\right)\right)
$$

where $A_{n}=\left\{a_{k}\right\}_{k=1}^{n}$ is any ray system of points that satisfies condition (1.1), $\left\{B_{k}\right\}_{k=1}^{n}$ is any collection of partially non-overlapping domains that satisfies condition (1.3), $a_{k} \in B_{k} \subset \overline{\mathbb{C}}$, and to describe all extremals $(k=\overline{1, n})$. 


\section{The case of open set}

Lemma 2.1. The function

$$
P(\tau)=\ln \sin \frac{\pi \tau}{2}
$$

is convex for $\tau \in(0,2)$.

The proof of the lemma can be found in the papers [20].

Theorem 2.1. Let $n \in \mathbb{N}, n \geq 2, \alpha \geq 0$. Then for all system points $A_{n}=\left\{a_{k}\right\}_{k=1}^{n}$, the satisfied condition (1.1) and condition

$$
\left(\left|a_{k}\right|-\left|a_{k+1}\right|\right)^{2}=4 \sin ^{2} \frac{\pi \sigma_{k}}{2}\left(1-\left|a_{k}\right|\left|a_{k+1}\right|\right), k=\overline{1, n},
$$

arbitrary open set $D$, the satisfied condition (1.2), $a_{k} \in D \subset \overline{\mathbb{C}}, k=\overline{1, n}$, be satisfied inequality

$$
\prod_{k=1}^{n}\left(\left|a_{k+1}-a_{k}\right|^{\alpha} \cdot r\left(D, a_{k}\right)\right) \leq\left(\frac{2^{\alpha+2}}{n} \cdot \sin ^{\alpha} \frac{\pi}{n}\right)^{n} \cdot \mathrm{T}\left(A_{n}\right) .
$$

The equality obtain in this inequality, when $D=\bigcup_{k=1}^{n} B_{k}$, where points $a_{k}$ and domains $B_{k}$ are, conformity, the poles and the circular domains of the quadratic differential

$$
Q(w) d w^{2}=-\frac{w^{n-2}}{\left(w^{n}-1\right)^{2}} d w^{2} .
$$

Proof. At once we will note that from the condition of unapplying follows that cap $\overline{\mathbb{C}} \backslash D>0$ and set $D$ possesses Green's generalized function $g_{D}(z, a)$, where

$$
g_{D}(z, a)=\left\{\begin{array}{l}
g_{D(a)}(z, a), z \in D(a), \\
0, z \in \overline{\mathbb{C}} \backslash \overline{D(a)}, \\
\lim _{\zeta \rightarrow z} g_{D(a)}(\zeta, a), \zeta \in D(a), z \in \partial D(a)
\end{array}\right.
$$

- Green's generalized function open set $D$ concerning a point $a \in D$, and $g_{D(a)}(z, a)$ - Green's function domain $D(a)$ concerning a point $a \in D(a)$. Further, we will use methods of works $[4,6,7]$. Sets we will consider $E_{0}=\overline{\mathbb{C}} \backslash D ; E\left(a_{k}, t\right)=\left\{w \in \mathbb{C}:\left|w-a_{k}\right| \leqslant t\right\}, \quad k=\overline{1, n}, n \geqslant 2, n \in \mathbb{N}$, $t \in \mathbb{R}_{+}$. The condenser we will enter into consideration for rather small $t>0$

$$
C\left(t, D, A_{n}\right)=\left\{E_{0}, E_{1}\right\},
$$


where

$$
E_{1}=\bigcup_{k=1}^{n} E\left(a_{k}, t\right)
$$

Capacity of the condenser $C\left(t, D, A_{2 n, 2 m-1}\right)$ is called as ( [4], [6])

$$
\operatorname{cap} C\left(t, D, A_{n}\right)=\inf \iint\left[\left(G_{x}^{\prime}\right)^{2}+\left(G_{y}^{\prime}\right)^{2}\right] d x d y,
$$

where an infimum undertakes on all continuous and to the lipschicevym in $\overline{\mathbb{C}}$ functions $G=G(z)$, such that $\left.G\right|_{E_{0}}=0,\left.G\right|_{E_{1}}=1$.

Let is named the module of condenser $C$, reverse the capacity of condenser

$$
|C|=[\operatorname{cap} C]^{-1}
$$

From a theorem $1[16]$ get

$$
\left|C\left(t, D, A_{2 n, 2 m-1}\right)\right|=\frac{1}{2 \pi} \cdot \frac{1}{n} \cdot \log \frac{1}{t}+M\left(D, A_{n}\right)+o(1), t \rightarrow 0,
$$

where

$$
M\left(D, A_{n}\right)=\frac{1}{2 \pi} \cdot \frac{1}{n^{2}} \cdot\left[\sum_{k=1}^{n} \log r\left(D, a_{k}\right)+2 \sum_{k \neq q} g_{D}\left(a_{k}, a_{q}\right)\right] .
$$

Function

$$
\zeta_{k}(w)=-i\left(e^{-i \arg a_{k}} w\right)^{\frac{1}{\sigma_{k}}}, \quad k=1,2, \ldots, n
$$

realizes univalent and conformal transformations of domain $M_{k}$ to the right half-plane $\operatorname{Re} \zeta>0$, for all $k=\overline{1, n}$.

From a formula (2.5) we receive the following asymptotic expressions

$$
\begin{gathered}
\left|\zeta_{k}(w)-\zeta_{k}\left(a_{m}\right)\right| \sim \frac{1}{\sigma_{k}}\left|a_{m}\right|^{\frac{1}{\sigma_{k}}-1}\left|w-a_{m}\right|, \\
w \rightarrow a_{m}, \quad k=1,2, \ldots, n, m=k, k+1 .
\end{gathered}
$$

It's obvious that

$$
\zeta_{k}\left(a_{k}\right)=-i\left|a_{k}\right|^{\frac{1}{\sigma_{k}}}, \zeta_{k}\left(a_{k+1}\right)=i\left|a_{k+1}\right|^{\frac{1}{\sigma_{k}}}, k=1,2, \ldots, n, a_{n+1}:=a_{1} .
$$

For any domain $\Delta \in \mathbb{C}$ the define $(\Delta)^{*}:=\{w \in \overline{\mathbb{C}}: \bar{w} \in \Delta\}$.

Let $\boldsymbol{\Omega}_{k}^{(1)}$ define connected component $\zeta_{k}\left(D \bigcap \bar{M}_{k}\right) \cup\left(\zeta_{k}\left(D \bigcap \bar{M}_{k}\right)\right)^{*}$, containing a point $\zeta_{k}\left(a_{k}\right)$, a $\boldsymbol{\Omega}_{k-1}^{(2)}-$ connected component 
$\zeta_{k-1}\left(D \bigcap \bar{M}_{k-1}\right) \bigcup\left(\zeta_{k-1}\left(D \bigcap \bar{M}_{k-1}\right)\right)^{*}$, containing a point $\zeta_{k-1}\left(a_{k}\right)$, $k=\overline{1, n}, \boldsymbol{\Omega}_{0}^{(2)}:=\boldsymbol{\Omega}_{n}^{(2)}$. It is clear, that $\boldsymbol{\Omega}_{k}^{(s)}$ generally speaking, domains are multiconnected domains, $k=\overline{1, n}, s=1,2$. Pair of domains $\boldsymbol{\Omega}_{k-1}^{(2)}$ and $\boldsymbol{\Omega}_{k}^{(1)}$ grows out of piece-dividing transformation open set $D$ concerning families $\left\{M_{k-1}, M_{k}\right\},\left\{\zeta_{k-1}, \zeta_{k}\right\}$ in point $a_{k}, k=\overline{1, n}, \bar{M}_{0}:=\bar{M}_{n}$, $\zeta_{0}:=\zeta_{n}$.

Let's consider condensers

$$
C_{k}\left(t, D, A_{n}\right)=\left(E_{0}^{(k)}, E_{1}^{(k)}\right)
$$

where

$$
E_{s}^{(k)}=\zeta_{k}\left(E_{s} \bigcap \bar{P}_{k}\right) \bigcup\left[\zeta_{k}\left(E_{s} \bigcap \bar{P}_{k}\right)\right]^{*},
$$

$k=\overline{1, n}, s=0,1,\left\{M_{k}\right\}_{k=1}^{n}$ - the system of corners corresponding to system of points $A_{n}$; operation $[A]^{*}$ compares to any the set $A \subset \overline{\mathbb{C}}$ a set, symmetric a set $A$ is relative unit circle $|w|=1$. From this it follows that to the condenser $C\left(t, D, A_{n}\right)$, at dividing transformation is relative $\left\{P_{k}\right\}_{k=1}^{n}$ and $\left\{\zeta_{k}\right\}_{k=1}^{n}$, there corresponds a set of condensers the system of corners corresponding to system of points $A_{n}$; operation $[A]^{*}$ compares to any the set $A \subset \overline{\mathbb{C}}$ a set, symmetric a set $A$ is relative unit circle $|w|=$ 1. From this it follows that to the condenser $C\left(t, D, A_{n}\right)$, at dividing transformation is relative $\left\{M_{k}\right\}_{k=1}^{n}$ and $\left\{\zeta_{k}\right\}_{k=1}^{n}$, there corresponds a set of condensers $\left\{C_{k}\left(t, D, A_{n}\right)\right\}_{k=1}^{n}$, symmetric relatively $\{z:|z|=1\}$. According to works $[4,16]$, we will receive

$$
\operatorname{cap} C\left(t, D, A_{n}\right) \geqslant \frac{1}{2} \sum_{k=1}^{n} \operatorname{cap} C_{k}\left(t, D, A_{n}\right)
$$

From here follows

$$
\left|C\left(t, D, A_{n}\right)\right| \leqslant 2\left(\sum_{k=1}^{n}\left|C_{k}\left(t, D, A_{n}\right)\right|^{-1}\right)^{-1} .
$$

The formula (2.3) gives a module asymptotics $C\left(t, D, A_{n}\right)$ at $t \rightarrow 0$, and $M\left(D, A_{n}\right)$ is the given module of a set $D$ relatively $A_{n}$. Using formulas (2.6) and that fact that a set $D$ meets the condition of unapplied in relation to the system of points $A_{n}$, for condensers we will receive similar asymptotic representations $C_{k}\left(t, D, A_{n}\right), k=\overline{1, n}$

$$
\left|C_{k}\left(t, D, A_{n}\right)\right|=\frac{1}{4 \pi} \log \frac{1}{t}+M_{k}\left(D, A_{n}\right)+o(1), t \rightarrow 0,
$$


where

$$
M_{k}\left(D, A_{n}\right)=\frac{1}{8 \pi} \cdot\left[\log \frac{r\left(\boldsymbol{\Omega}_{k}^{(1)}, \zeta_{k}\left(a_{k}\right)\right)}{\frac{1}{\sigma_{k}}\left|a_{k}\right|^{\frac{1}{\sigma_{k}}-1}}+\log \frac{r\left(\boldsymbol{\Omega}_{k-1}^{(2)}, \zeta_{k-1}\left(a_{k}\right)\right)}{\frac{1}{\sigma_{k-1}}\left|a_{k}\right|^{\frac{1}{\sigma_{k-1}}-1}}\right] .
$$

By means of (2.10), we receive

$$
\begin{aligned}
& \left|C_{k}\left(t, D, A_{n}\right)\right|^{-1}=\frac{4 \pi}{\log \frac{1}{t}} \cdot\left(1+\frac{4 \pi}{\log \frac{1}{t}} M_{k}\left(D, A_{n}\right)+o\left(\frac{1}{\log \frac{1}{t}}\right)\right)^{-1} \\
& =\frac{4 \pi}{\log \frac{1}{t}}-\left(\frac{4 \pi}{\log \frac{1}{t}}\right)^{2} M_{k}\left(D, A_{n}\right)+o\left(\left(\frac{1}{\log \frac{1}{t}}\right)^{2}\right), t \rightarrow 0
\end{aligned}
$$

Further, from (2.11), follows that

$$
\begin{gathered}
\sum_{k=1}^{n}\left|C_{k}\left(t, D, A_{n}\right)\right|^{-1} \\
=\frac{4 \pi n}{\log \frac{1}{t}}-\left(\frac{4 \pi}{\log \frac{1}{t}}\right)^{2} \cdot \sum_{k=1}^{n} M_{k}\left(D, A_{n}\right)+o\left(\left(\frac{1}{\log \frac{1}{t}}\right)^{2}\right), \quad t \rightarrow 0 .
\end{gathered}
$$

In turn, allows (2.12) to receive the following asymptotic representation

$$
\begin{gathered}
\left(\sum_{k=1}^{n}\left|C_{k}\left(t, D, A_{n}\right)\right|^{-1}\right)^{-1} \\
=\frac{\log \frac{1}{t}}{4 \pi n} \cdot\left(1-\frac{4 \pi}{n \log \frac{1}{t}} \cdot \sum_{k=1}^{n} M_{k}\left(D, A_{n}\right)+o\left(\frac{1}{\log \frac{1}{t}}\right)\right)^{-1} \\
=\frac{\log \frac{1}{t}}{4 \pi n}+\frac{1}{n^{2}} \cdot \sum_{k=1}^{n} M_{k}\left(D, A_{n}\right)+o(1), \quad t \rightarrow 0 .
\end{gathered}
$$

Inequalities, (2.8) and (2.9) taking into (2.3) and (2.13) allow to notice that

$$
\frac{1}{2 \pi} \cdot \frac{1}{n} \cdot \log \frac{1}{t}+M\left(D, A_{n}\right)+o(1) \leqslant \frac{\log \frac{1}{t}}{2 \pi n}+\frac{2}{n^{2}} \cdot \sum_{k=1}^{n} M_{k}\left(D, A_{n}\right)+o(1) .
$$

From (2.14) at $t \rightarrow 0$ we receive that

$$
M\left(D, A_{n}\right) \leqslant \frac{2}{n^{2}} \cdot \sum_{k=1}^{n} M_{k}\left(D, A_{n}\right) .
$$


Formulas (2.4), (2.10) and (2.15) lead to the following expression

$$
\begin{gathered}
\frac{1}{2 \pi} \cdot \frac{1}{n^{2}} \cdot\left[\sum_{k=1}^{n} \log r\left(D, a_{k}\right)+2 \sum_{k \neq q} g_{D}\left(a_{k}, a_{q}\right)\right] \\
\leq \frac{1}{4 \pi n^{2}} \cdot\left[\sum_{k=1}^{n} \log \frac{r\left(\boldsymbol{\Omega}_{k}^{(1)}, \zeta_{k}\left(a_{k}\right)\right)}{\frac{1}{\sigma_{k}}\left|a_{k}\right|^{\frac{1}{\sigma_{k}}-1}}+\sum_{k=1}^{n} \log \frac{r\left(\boldsymbol{\Omega}_{k-1}^{(2)}, \zeta_{k-1}\left(a_{k}\right)\right)}{\frac{1}{\sigma_{k-1}}\left|a_{k}\right|^{\frac{1}{\sigma_{k-1}}-1}}\right] .
\end{gathered}
$$

Thus, taking into (2.1), we receive

$$
\begin{gathered}
\prod_{k=1}^{n}\left(\left|a_{k+1}-a_{k}\right|^{\alpha} \cdot r\left(D, a_{k}\right)\right) \leq 2^{n \alpha} \cdot \prod_{k=1}^{n} \frac{\sigma_{k}\left|a_{k}\right|}{\left|a_{k}\right|^{\frac{1}{2 \sigma_{k}}} \cdot\left|a_{k}\right|^{\frac{1}{2 \sigma_{k-1}}}} \\
\times \prod_{k=1}^{n} \sin ^{\alpha} \frac{\pi \sigma_{k}}{2} \cdot \prod_{k=1}^{n}\left(r\left(\boldsymbol{\Omega}_{k}^{(1)}, \zeta_{k}\left(a_{k}\right)\right) \cdot r\left(\boldsymbol{\Omega}_{k}^{(2)}, \zeta_{k}\left(a_{k+1}\right)\right)\right)^{\frac{1}{2}} .
\end{gathered}
$$

The equality obtain in this inequality, when points $a_{k}$ and domains $B_{k}$ are, conformity, the poles and the circular domains of the quadratic differential

$$
Q(\zeta) d \zeta^{2}=\frac{d \zeta^{2}}{\left(\zeta^{2}+1\right)^{2}} .
$$

Using the Lemma 2.1 that the function $\alpha \ln \sin \frac{\pi \sigma_{k}}{2}$, is convex for $\sigma_{k} \in(0 ; 2), \alpha \geq 0$. Hence, when $\sigma_{k} \in(0 ; 2)$, then

$$
\frac{\alpha}{n} \cdot \sum_{k=1}^{n} \ln \sin \frac{\pi \sigma_{k}}{2} \leq \alpha \ln \sin \left(\frac{\pi}{2} \cdot \frac{1}{n} \sum_{k=1}^{n} \sigma_{k}\right) .
$$

Given that

$$
\sum_{k=1}^{n} \sigma_{k}=2
$$

we obtain

$$
\prod_{k=1}^{n} \sin ^{\alpha} \frac{\pi \sigma_{k}}{2} \leq \sin ^{n \alpha} \frac{\pi}{n}
$$

The equality obtain in this inequality, if and only if

$$
\sigma_{1}=\sigma_{2}=\ldots=\sigma_{n}=\frac{2}{n} .
$$

Then from (2.16) using formulas (2.18) it is received the following ratio

$$
\prod_{k=1}^{n}\left(\left|a_{k+1}-a_{k}\right|^{\alpha} \cdot r\left(B_{k}, a_{k}\right)\right) \leq\left(\frac{2^{\alpha+2}}{n}\right)^{n} \cdot \mathrm{T}\left(A_{n}\right) \cdot \sin ^{n \alpha} \frac{\pi}{n} .
$$


The equality obtain in this inequality, when points $a_{k}$ and domains $B_{k}$ are, conformity, the poles and the circular domains of the quadratic differential (2.2). It is derived from the square of the quadratic differential (2.17) conversion using

$$
\zeta=-i w^{\frac{n}{2}}
$$

\section{The case of partially non-overlapping domains}

Theorem 3.1. Let $n \in \mathbb{N}, n \geq 2, \alpha \geq 0$. Then for all system points $A_{n}=\left\{a_{k}\right\}_{k=1}^{n}$, the satisfied condition (1.1) and condition (2.1), arbitrary system partially non-overlapping domains $B_{k}$, the satisfied condition (1.3), $a_{k} \in B_{k} \subset \overline{\mathbb{C}}, k=\overline{1, n}$, be satisfied inequality

$$
\prod_{k=1}^{n}\left(\left|a_{k+1}-a_{k}\right|^{\alpha} \cdot r\left(B_{k}, a_{k}\right)\right) \leq\left(\frac{2^{\alpha+2}}{n} \cdot \sin ^{\alpha} \frac{\pi}{n}\right)^{n} \cdot \mathrm{T}\left(A_{n}\right) .
$$

The equality obtain in this inequality, where points $a_{k}$ and domains $B_{k}$ are, conformity, the poles and the circular domains of the quadratic differential (2.2).

Proof. In case of partially non-overlapping domains, the open set is entered by a representation (1.3), which satisfies (1.2). From here, we have

$$
B_{k} \subset D, \quad k=\overline{1, n} .
$$

From (3.1), we receive, using results works $[5,6,21]$

$$
r\left(B_{k}, a_{k}\right) \leq r\left(D, a_{k}\right), \quad k=\overline{1, n} .
$$

Multiplying inequalities (3.2), we draw a conclusion

$$
\prod_{k=1}^{n}\left(\left|a_{k+1}-a_{k}\right|^{\alpha} \cdot r\left(B_{k}, a_{k}\right)\right) \leq \prod_{k=1}^{n}\left(\left|a_{k+1}-a_{k}\right|^{\alpha} \cdot r\left(D, a_{k}\right)\right) .
$$

We receive an end result using the theorem 2.1.

\section{References}

[1] M. A. Lavrent'ev, On the theory of conformal mappings // Tr. Fiz.-Mat. Inst. Akad. Nauk SSSR, 5 (1934), 159-245. 
[2] G. M. Goluzin, Geometric theory of functions of a complex variable, Nauka, Moscow, 1966.

[3] G. P. Bakhtina, Variational methods and quadratic differentials in problems for disjoint domains, PhD thesis, Kiev, 1975.

[4] A. K. Bakhtin, G. P. Bakhtina, Yu. B. Zelinskii, Topological-algebraic structures and geometric methods in complex analysis, Inst. Math. NAS Ukraine, Kiev, 2008.

[5] V. N. Dubinin, Separating transformation of domains and problems of extremal division // Zap. Nauchn. Sem. Leningrad. Otdel. Mat. Inst. Ros. Akad. Nauk, 168 (1988), 48-66.

[6] V. N. Dubinin, Method of symmetrization in the geometric theory of functions of a complex variable // Usp. Mat. Nauk, 49 (1994), No. 1, 3-76.

[7] A. K. Bakhtin, Inequalities for the inner radii of nonoverlapping domains and open sets // Ukr. Math. J., 61 (2009), No. 5, 716-733.

[8] A. K. Bakhtin, A. L. Targonskii, Extremal problems and quadratic differential // Nonlin. Oscillations, 8 (2005), No. 3, 296-301.

[9] A. L. Targonskii, Extremal problems of partially nonoverlapping domains on a Riemann sphere // Dop. NAN Ukr., 9 (2008), 31-36.

[10] A. Targonskii, Extremal problems on the generalized ( $n$; d)-equiangular system of points // An. St. Univ. Ovidius Constanta, 22 (2014), No. 2, 239-251.

[11] A. Targonskii, Extremal problem (2n; 2m-1)-system points on the rays // An. St. Univ. Ovidius Constanta, 24 (2016), No. 2, 283-299.

[12] A. L. Targonskii, Extremal problems for partially non-overlapping domains on equiangular systems of points // Bull. Soc. Sci. Lett. Lodz, 63 (2013), No. 1, $57-63$.

[13] A. Targonskii, I. Targonskaya, On the One Extremal Problem on the Riemann Sphere // International Journal of Advanced Research in Mathematics, 4 (2016), $1-7$.

[14] A. K. Bakhtin, A. L. Targonskii, Generalized ( $n$, d)-ray systems of points and inequalities for nonoverlapping domains and open sets // Ukr. Math. J., 63 (2011), No. 7, 999-1012.

[15] A. K. Bakhtin, A. L. Targonskii, Some extremal problems in the theory of nonoverlapping domains with free poles on rays // Ukr. Math. J., 58 (2006), No. 12, 1950-1954.

[16] V. N. Dubinin, Asymptotic representation of the modulus of a degenerating condenser and some its applications // Zap. Nauchn. Sem. Peterburg. Otdel. Mat. Inst., 237 (1997), 56-73.

[17] V. N. Dubinin, Capacities of condensers and symmetrization in geometric function theory of complex variables, Dal'nayka, Vladivostok, 2009. 
[18] A. Targonskii, An extremal problem for the nonoverlapping domains // Ukr. Math. Bull., 14 (2017), No. 1, 126-134; transl. Journal of Mathematical Sciences, 227 (2017), No. 1, 98- 104.

[19] A. Targonskii, I. Targonskaya, Extreme problem for partially nonoverlapping domains on a Riemann sphere // Ukr. Math. Bull., 15 (2018), No. 1, 94-102; transl. Journal of Mathematical Sciences, 235 (2018), No. 1, 74-80.

[20] A. L. Targonskii, About one extremal problem for projections of the points on unit circle // Ukrainian Mathematical Bulletin, 15 (2018), No. 3, 418-430.

[21] W. K. Hayman, Multivalent functions, Cambridge University, Cambridge, 1958.

[22] J.A. Jenkins, Univalent functions and conformal mapping, Springer, Berlin, 1958.

\section{CONTACT INFORMATION}

Andrei Leonidovich I. Franko Zhitomir State University, Targonskii Zhitomir, Ukraine

E-Mail: targonsk@zu.edu.ua

Irina Igorevna

Targonskaya

Katya Vaschenko
I. Franko Zhitomir State University, Zhitomir, Ukraine

E-Mail: targonsk@zu.edu.ua

I. Franko Zhitomir State University, Zhitomir, Ukraine E-Mail: katyavaschenko@ukr.net 